\title{
Frequency and distribution of rare electrophoretic mobility variants in a population of human newborns in Ann Arbor, Michigan
}

\author{
H. W. MOHRENWEISER*, K. H. WURZINGER AND J. V. NEEL \\ Department of Human Genetics, University of Michigan Medical School, Ann Arbor, MI 48109
}

\section{SUMMARY}

We have summarized the frequency and distribution of the rare variants encountered during the screening of 258815 allele products, the products of 51 different loci, in 3242 predominantly Caucasian ( $88 \%$ ) newborns. Seventy-nine different rare variants, representing 187 occurrences, were identified. Almost $60 \%$ (46 of 79) of the rare variants occurred as singletons while another $20 \%$ were seen in two unrelated individuals. No rare variants were detected at 18 loci while no variants, either rare or polymorphic, were detected at 14 loci. More rare variants were identified at loci that were classified as polymorphic and also at loci where the gene products exist as a monomer. A positive relationship was observed between variant frequency, either classes or copies, and subunit molecular mass.

\section{INTRODUCTION}

Each new study of the level of genetic variation among individuals/populations highlights the diversity in the human gene pool. The variation observed is of two general classes. The first is variation which is polymorphic, that is, a few variants at a locus are widely distributed among many individuals in a population. In contrast, rare variants are, by definition, detected among a relatively few individuals in the population, the usual definition being that a phenotype with a frequency of less than $2 \%$ is considered a rare variant. The latter are more likely to represent the spectrum of relatively recent mutational events than are the polymorphisms, which for a variety of reasons represent the few survivors from among many mutational events occurring in the more remote past.

The number of studies that have surveyed, by electrophoretic techniques, a wide range of loci, including loci expected to be monomorphic, has been quite limited (Harris et al. 1974; Neel et al. 1978; Neel et al. 1980). Within the context of a pilot study concerned with the feasibility of using electrophoretic techniques to detect germinal mutations in a human population (Neel et al. 1987) we have accumulated, during the last 10 years, an extensive data base useful for an analysis of rare variant frequency at 51 loci. The loci studied were selected because of the availability of electrophoretic techniques permitting high resolution of their gene products rather than on the bases of anticipated variation, biological function or other restrictive criteria. This data base is especially suited for addressing questions regarding rare variant frequencies in a human population and the extent of variation in rare variant frequency among loci of the human genome.

* Current address: Biomedical Sciences Division, Lawrence Livermore National Laboratory, Livermore, CA 94550 
Cord blood samples were collected from 3456 newborns and their parents at Women's Hospital at the Cniversity of Michigan during the period of this study. To the extent possible, samples were collected from all newborns where informed consent and a blood sample from both parents could be obtained. In our previous publication on this series (Neel et al. 1987), one of each pair of identical twins was excluded but otherwise all siblings were allowed to remain in the series as each is an independent test of mutation. This resulted in 277747 locus tests. Seven percent of the samples were from siblings, including non-identical twins, as determined by examination of mothers hospital identification numbers. For the present series, to eliminate non-binomial variation in statistical tests, only the first child in a family has been included in this data base. which reduces the number of newborns in the series to 3242 and the number of locus tests to 258815 . The population is $88 \%$ Caucasian and $7 \%$ Black while the remaining $5 \%$ of the population includes individuals of mixed or unknown racial origin and a very limited number of individuals with other racial backgrounds. The number of unrelated newborns studied ranges from 2827 for PGM1 to 632 for $\mathrm{BG} 7 \mathrm{~S}$ in the Caucasian sample and from 41 for $\mathrm{BGTS}$ to 242 for several loci in the Black sample. Differences in the number of determinations (individuals studied) reflect the sequential addition of new loci as electrophoretic techniques were developed.

A question arises as to the definition of a 'rare variant' when one is screening representatives of different ethnic groups. This is especially a consideration when, as in this series, some 20 $30 \%$ of the gene pool of the American Black group is known to be derived from the Caucasoid group, with a more limited gene flow in the other direction (summary in Reed, 1969). In the present paper. we have elected not to count as a rare variant in either group, any allele with a frequency greater than 0.01 in either of the two groups. This convention, however, does influence the direct comparison of our average variant frequencies with those of other investigators, who also face the problem of ethnic admixture, albeit usually in a less pronounced form. Accordingly the impact of this convention on the estimated variant frequency will also be discussed.

\section{METHODS}

Electrophoretic methods for most of the proteins studied have been previously described (Neel et al. 1980 ; Barrantes et al. 1982 ; Naidu et al. 1985; Long et al. 1986). The electrophoretic system for inosine triphosphatase (ITPA), enolase (ENO1), diaphorase (DIA) and acetaldehyde dehydrogenase (ALDH) was the FUM/GPT buffer system described by Long et al. (1986). ITPA. DIA and ENO1 were stained as described by Harris \& Hopkinson (1976) while ALDH was stained as described by Pearse (1972). Prealbumin (PALB) was typed on a $7 \%$ acrylamide gel with a gel buffer of 1.25 $\mathrm{m}$-Tris- $\mathrm{HCl}, \mathrm{pH} \mathrm{9 \cdot 1}$, and a tank buffer of 0.04 $\mathrm{m}$-Tris glycine, $\mathrm{pH} \mathrm{8.5.}$ PALB was localized by staining with Coomassie Blue. 7-S beta globulin (BG7S) was typed as described by Haupt \& Böhm (1977). The method for apolipoprotein A (APOA1) was modified from Haupt \& Böhm (1977). The first step was electrophoresis of plasma, following treatment with 'Triton X-100 and cetyltrimethylammonium bromide, on agarose gels with a Tris glycine buffer. The second step involved isoelectric focusing ( $\mathrm{pH} \mathrm{4.0-6.5}$ gradient) of the proteins of the agarose gel section containing the APOA1 on a polyacrylamide gel containing $8 \mathrm{~m}$ urea. The 
APOA1 bands were visualized by staining with Coomassie Blue. Sample storage and preparation of haemolysates were as described by Neel et al. (1980).

\section{RESULTS}

The data on number of determinations, classes of rare variants, total number of copies of rare variants and number of copies of each class of rare variants for Caucasians and Blacks are in Table $1 a$ and $1 b$, respectively. Tables $1 a, b$ also present the allele counts (multiply determinations by two except for sex linked loci) for the polymorphic alleles in the major ethnic groups. The genetic nature of all rare and low frequency polymorphic variants was determined by establishing the occurrence of the variant in one of the child's parents. Although the electrophoretic mobilities of the variants have been rigorously compared, using standard techniques, to establish the minimum number of electrophoretic classes, no systematic attempt has been made to make direct comparison of these variants with those reported by others. Therefore, the variants have not been assigned names unless they have been the subject of other publications concerned with their biochemical characteristics. These would include variants at the following loci : CRPL, Mohrenweiser \& Decker, 1982; GOTS, Wurzinger \& Mohrenweiser, 1982 ; TPI, Asakawa \& Mohrenweiser, 1982; TF, Fujita et al. 1985; GPI, Mohrenweiser et al. 1987 ; $G 6 P D$, Mohrenweiser \& Fielek, in preparation. The allele frequencies at the polymorphic loci are very similar to the previously published frequencies for these two ethnic groups in the United States (Mourant et al. 1976; Silver, 1982; Tills et al. 1983).

In the Caucasian sample, 64 different products of rare alleles, distributed among 154 individuals, were identified from a total of 114560 determinations (1.3 variants/1000 determinations). Seventeen different products of rare alleles, occurring in 27 copies (individuals), were detected in 9634 determinations among the Black sample ( $2 \cdot 8$ variants $/ 1000$ determinations). The frequency of different rare variants is higher in the Black sample (1.8 variant classes $/ 1000$ determinations $)$ than in the Caucasian sample $(0 \cdot 6$ variant classes $/ 1000$ determinations) $\left(\chi^{2}=12.9\right.$, D.F. $\left.=1, P<0.01\right)$. In the total (pooled) sample, 79 different rare variants occurred in a total of 187 copies in the screening of 258815 alleles (1.4 variants/1000 determinations). If one does not exclude those variants which were found to be polymorphic in the other ethnic group, then the variant frequencies are as follows: in the Caucasian sample, there were 72 different rare allele products distributed among 255 individuals, resulting in a frequency of 0.6 variant classes/1000 determinations; in the Black population, there were 18 different rare allele products distributed among 28 individuals with a resulting frequency of 1.8 variant classes / 1000 determinations. Thus, the higher number of different rare alleles in Blacks is independent of the method of defining rare variants. By contrast, the number of variants encountered becomes $2 \cdot 2 / 1000$ determinations for Caucasians and 2.9/1000 determinations for Blacks. Eighty percent of the increase in number of variants in the Caucasian group is contributed by the CRPL*MI and PEPD*2 alleles, which along with the TF*DCHI variant, account for $45 \%$ of all of the rare variants in the Caucasian group.

Several of the alleles designated in Tables $1 a, b$ have been subdivided by utilizing additional electrophoretic or thermostability techniques (Wurzinger \& Mohrenweiser, 1982; Fujita et al. 1985). Therefore, the estimate of 79 different rare alleles is an absolute minimum estimate as this estimate is based upon variant classes identified by the original electrophoretic procedures 

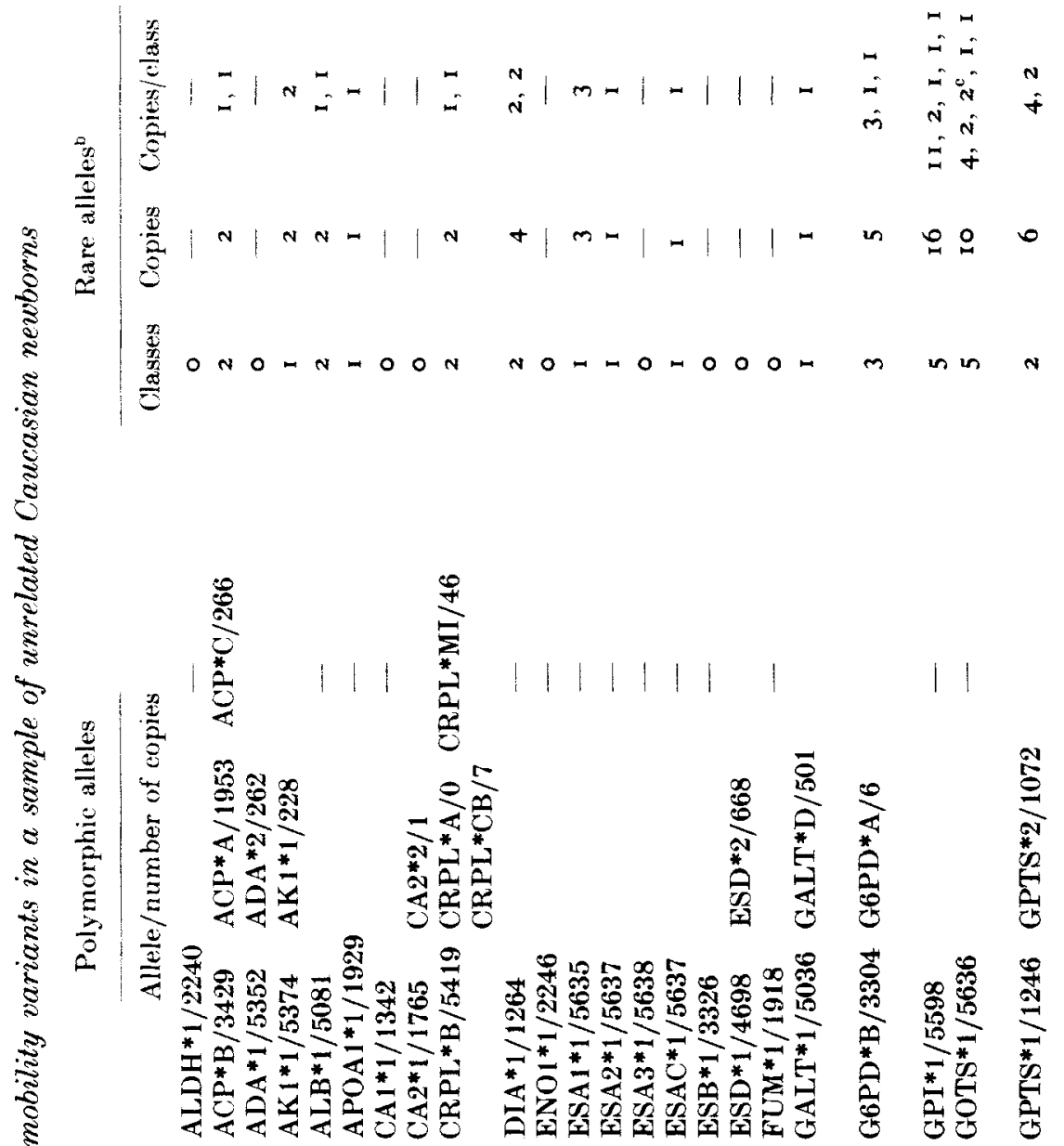

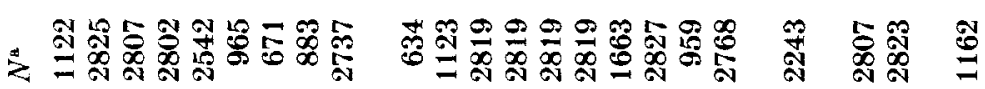

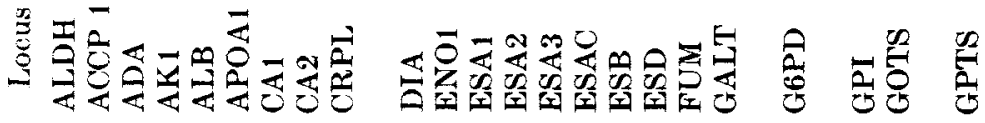

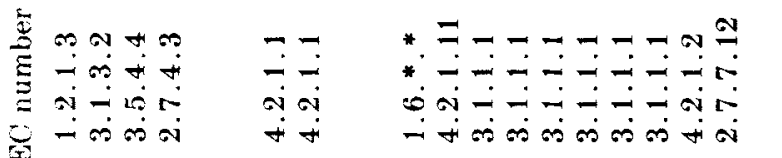
焉
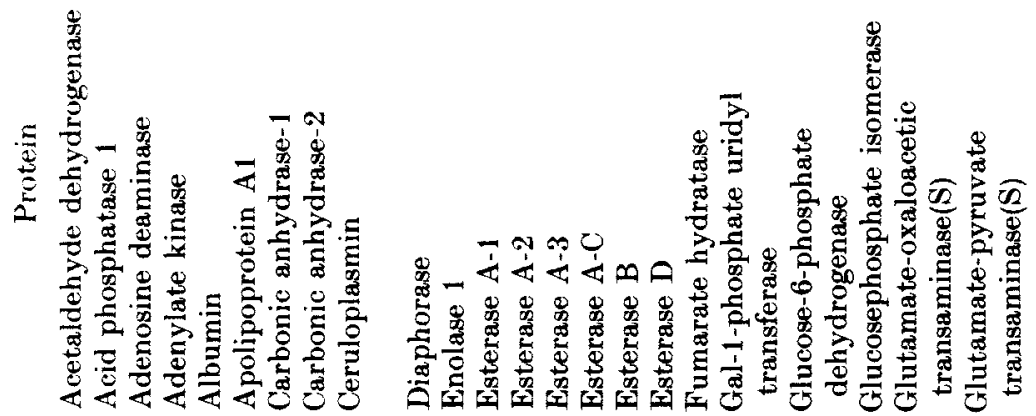


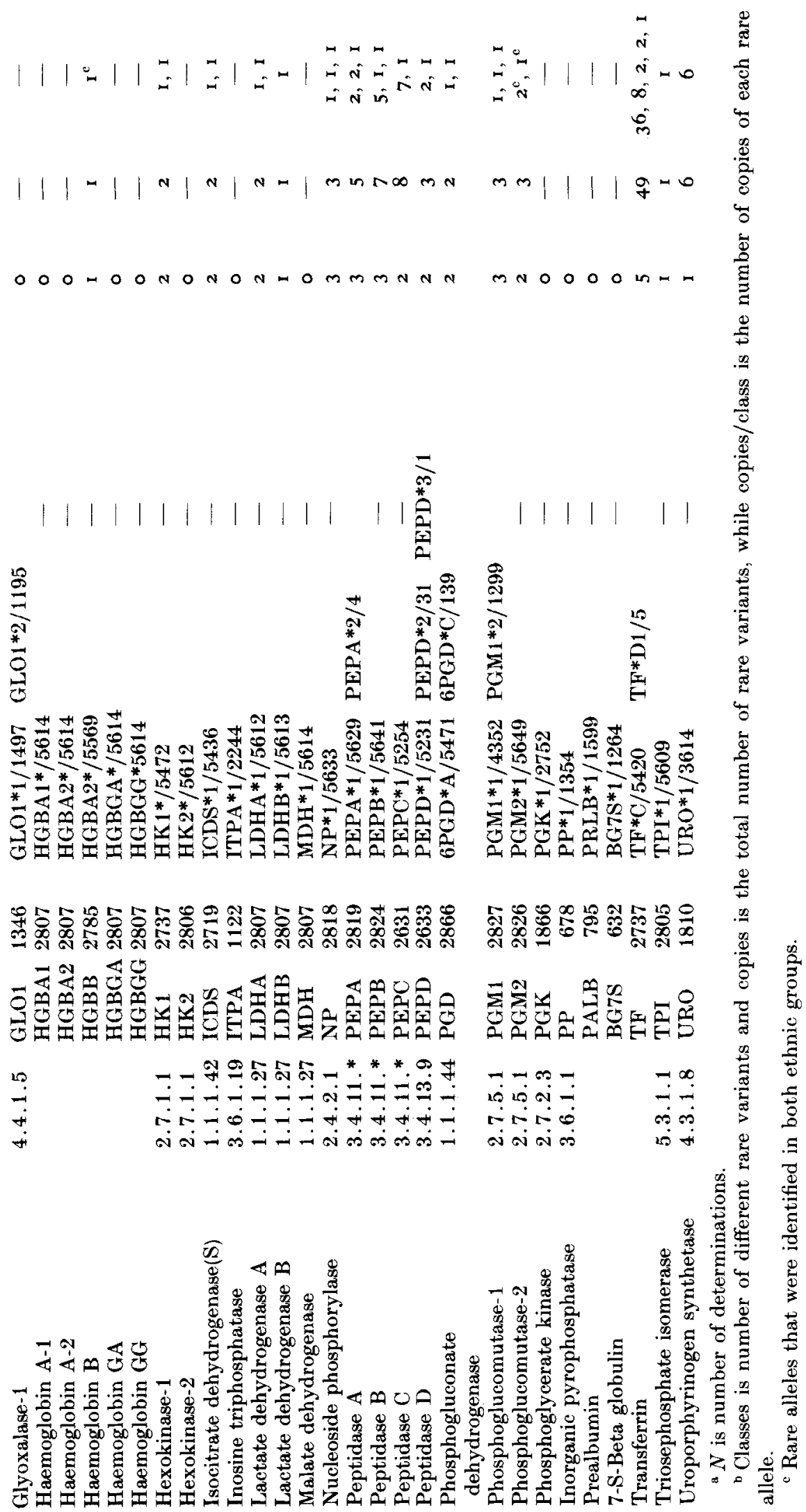


Table $1 b$. Frequency of rare electrophoretic mobility variants in a sample of unrelated Black newborns

Polymorphic alleles

Lorus $\quad N^{a}$

$\begin{array}{lr}\text { ALDH } & 86 \\ \text { ACP } & 24 \\ \text { ADA } & 238 \\ \text { AK } & 240 \\ \text { ALB } & 206 \\ \text { APOA1 } & 68 \\ \text { CA1 } & 73 \\ \text { CA2 } & 93 \\ \text { CRPL } & 23\end{array}$

DIA

ENO1

ESA1

ESA2

ESA3

ESAC

ESB

ESD

FUM

GALT

G6PD

GPI

GOTS

GPTS

GLO1

HGBA1

HGBA2

HGBB

HGBGA 238

HGBGG 238

HK 1

HK2

ICDS

ITPA

IDHA

LDHB

$\mathrm{MDH}$

NP

PEPA

PEPB

PEPC

PEPI)

PGD

PGM1

PGM2

PGK

PP

PALB

BG7S

TF

TPI

URO

$N^{a}$

Allele/number of copies

ALDH*1/172

$\mathrm{ACP} 1 * \mathrm{~B} / 371 \mathrm{ACP} 1 * \mathrm{~A} / 103 \mathrm{ACP} 1 * \mathrm{C} / 5$

ADA*1/471 ADA*2/5

AK $1 * 1 / 475$ AK $1 * 2 / 1$

ALB* $1 / 412$

APOA 1*1/134

CA1*1/144

CA2*1/177 CA2*2/9

CRPL*B/430 CRPL*A/18 CRPL*M1/6

$\mathrm{CRPL} * \mathrm{CB} / 5$

DIA*1/86

ENO1*1/172

$\operatorname{ESA1} 1 / 479$

$\operatorname{ESA1} * 1 / 480$

FSA3*1/480

ESAC $* 1 / 480$

$\mathrm{ESB}^{*} 1 / 258$

$\mathrm{ESD}^{* 1 / 439} \mathrm{ESD}^{* 2 / 41}$

FUM*1/140

GALT*1/454 GALT*D/14

$\mathrm{G} 6 \mathrm{PD}{ }^{*} \mathrm{~B} / 205 \quad \mathrm{G} 6 \mathrm{PD}^{*} \mathrm{~A} / 50$

GPI*1/476

GOTS*1/477

GPTS*1/128 GPTS*2/47

GLO1*1/177 GLO1*2/69

HGBA1*1/473

HGBA2*1/476

$\mathrm{HGBB}^{*} 1 / 473$

HGBGA*1/476

HGBGG*1/476

HK $1 * 1 / 466$

HK2*1/478

ICDS*1/454

ITPA*1/172

LDHA*1/472

LDHB*1/476

$\mathrm{MDH} * 1 / 476$

$N P * 1 / 482$

PEPA*1/445

PEPB*1/483

$P E P C * 1 / 441$

PEPD*/433 PEPD*2A /8 PEPD*2B $/ 5$

$\mathrm{PGD} * \mathrm{~A} / 461 \quad \mathrm{PGD}{ }^{*} \mathrm{C} / 13$

PGM1*1/396 PGM1*2/88

PGM2*1/482

PGK*1/206

$P P^{*} 1 / 144$

PALB*1/98

BG7S*1/82

$\mathrm{TF} * \mathrm{C} / 450 \quad \mathrm{TF}^{*} \mathrm{D} 1 / 12$

TPI*I/476

$\mathrm{URO} * 1 / 354$
Rare alleles

Classes Copies Copies/class

\footnotetext{
Footnotes are the same as in Table $1 a$.
} 
Table 2. Frequency distribution of classes of variants/locus

\begin{tabular}{|c|c|c|c|c|c|c|}
\hline \multirow[b]{3}{*}{$\begin{array}{l}\text { Variant } \\
\text { classes/ } \\
\text { locus }\end{array}$} & \multicolumn{6}{|c|}{ Number of occurrences } \\
\hline & \multicolumn{3}{|c|}{ Total alleles ${ }^{\mathrm{a}}$} & \multicolumn{3}{|c|}{ Rare alleles } \\
\hline & $\mathrm{CA}^{\mathrm{b}}$ & $B L^{e}$ & $\begin{array}{c}\text { Pooled }^{\mathrm{d}} \\
\text { total }\end{array}$ & $\mathrm{CA}^{\mathrm{b}}$ & $B L^{c}$ & $\begin{array}{c}\text { Pooled }^{\mathrm{d}} \\
\text { total }\end{array}$ \\
\hline 0 & - & - & - & 21 & 37 & I 8 \\
\hline I & 17 & 25 & $\mathrm{I} 4$ & 10 & I I & 10 \\
\hline 2 & I 2 & 20 & I 3 & I 2 & 2 & 9 \\
\hline 3 & 9 & 3 & 8 & 5 & I & 4 \\
\hline 4 & 4 & I & 4 & 0 & 0 & 8 \\
\hline 5 & 6 & 2 & 4 & 3 & 0 & I \\
\hline 6 & 2 & 0 & 4 & 0 & 0 & 0 \\
\hline 7 & I & 0 & 3 & 0 & 0 & I \\
\hline 8 & 0 & 0 & I & 0 & 0 & 0 \\
\hline \multicolumn{7}{|c|}{$\begin{array}{l}{ }^{2} \text { Rare plus polymorphic alleles. } \\
{ }^{\mathrm{C}} \mathrm{CA}=\text { Caucasian. } \\
{ }^{\mathrm{B}} \mathrm{BL}=\text { Black. }\end{array}$} \\
\hline
\end{tabular}

rather than subdivisions identified during subsequent studies to characterize some of the variants. This characterization provides insight into the level of microheterogeneity and is similar to the results from many similar studies designed to subdivide variants with apparently similar electrophoretic mobilities. Note that the number of variants identified differs from the data of Neel et al. (1987) because only unrelated (non-sibling) newborns have been included in the current sample.

The distribution of classes of variants, both total, rare plus polymorphic, and rare variants per locus is in Table 2. In the Caucasian sample, 17 of the 51 loci are monomorphic, that is, neither rare nor polymorphic variants were observed, while for 4 additional loci, $A D A, C A I I$, $E S D$ and $G L O I$, polymorphic but not rare mobility variants were detected. Rare variants were thus not detected at 30 of 51 loci. The average number of determinations at the 21 loci where rare variants were not detected was somewhat less $(\bar{x}=1772)$ than at the other 30 loci $(\bar{x}=$ 2573). The maximum number of alleles (rare plus polymorphic) at any locus was 7 for $T F$ in the Caucasian sample. In the combined sample, GOT exhibited 7 different rare alleles.

Most of the rare variants were recovered as singletons. Forty-one of the $64(64 \%)$ different rare allele products detected in the Caucasian sample occurred as a single copy while two copies were detected for another $13(20 \%)$ allele products (Table 3). In the total data set, 46 of the 79 $(58 \%)$ rare variants occurred only once and another $17(21 \%)$ twice.

The distribution of allele frequencies for the 133 gene products, including the most common variant, at each locus identified in the Caucasian sample is presented in Table 4. Sixteen monomorphic loci are included in the tabulation. Thirty-eight alleles have frequencies of less than 0.0002 which corresponds to a single copy in approximately 2700 determinations per locus. Allele frequencies of $0.0003-0.0005$ generally reflect two copies of an allele in 2700 determinations. At the other extreme, nineteen alleles, at nine loci, were encountered in polymorphic frequencies $(0 \cdot 01-0 \cdot 99)$. It should be noted that for 31 of the 51 loci studied, more than 2700 determinations were completed while $2000-2700$ determinations were completed for 
Table 3. Frequency distribution of copies of rare alleles per variant class

$\begin{array}{ccc}\begin{array}{c}\text { Number of } \\ \text { copies/rare } \\ \text { allele }\end{array} & \begin{array}{c}\text { Number of } \\ \text { occurrences }\end{array} \\ \text { I } & \text { Caucasian } & \begin{array}{c}\text { Pooled } \\ \text { total }\end{array} \\ 2 & 4 \text { I } & 46 \\ 3 & \text { I } & 17 \\ 4 & 2 & 6 \\ 5 & 2 & 2 \\ 6 & \text { I } & \text { I } \\ 7 & \text { I } & 2 \\ 8 & \text { I } & 2 \\ 9 & 0 & \text { I } \\ \text { I 1 } & \text { I } & \text { I } \\ \text { I 3 } & 0 & 0 \\ 36 & \text { I } & \text { 1 } \\ \text { s Includes all first borns, } & \text { irrespective of ethnicity. }\end{array}$

five other loci. For only 12 loci were less than 1400 individuals studied and the minimum sample size for any protein studied in the Caucasian series was 632 individuals. The units of this tabulation are of course sample size dependent and most such tabulations are based on smaller sample sizes, thus curtailing the lower limit rather sharply. Even within this sample, the varying number of determinations per locus is a complication. Thus, we have tabulated separately the 12 loci where less than 1400 unrelated individuals were studied. The number of rare variant classes does not differ significantly between the two groups, being 0.4 rare variant classes per 1000 determinations for the $<1400$ group and 0.5 for the $>1400$ group, but the sample-size-imposed constraint on the observed variant frequencies is obvious.

Several different comparisons of allelic frequencies are in Table 5. Among the proteins studied for electrophoretic mobility variants, 20 are monomers, (ALDH, ACP1, ADA, AK1, ALB, APOA1, CA1, CA2, CRPL, DIA, ESB, HK1, HK2, PEPB, PEPC, PGM1, PGM2, PGK, TF and URO) of which 13 are monomorphic and 7 are polymorphic. Thirty-one proteins occur as homoor heteropolymers, of which 23 are monomorphic and 8 are polymorphic. Rare variants were not detected at 20 loci of which 14 (of 31 ) were polymeric proteins and 6 (of 20 ) were monomers. The greater variability of the monomers is further reflected in a higher number of both total alleles and rare alleles per 1000 alleles and also more total copies of rare alleles than for proteins which exist as polymers. Although a pattern seems to exist, none of the differences are significant at a $P<0 \cdot 20$.

At loci classified as polymorphic (at least two alleles exist at a frequency greater than $0 \cdot 01$ ), the number of rare variant classes is higher than at monomorphic loci $\left(\chi^{2}=2 \cdot 5\right.$, D.F. $=1, P<$ $0 \cdot 15$ ). A problem exists in a similar comparison of the number of copies of rare alleles as 36 copies of a single transferrin variant were identified. This frequency is almost three times the number of variants encountered for the next most numerous rare variant. The total number of rare variant copies is higher at polymorphic loci if all of the data are utilized but the number of copies of rare alleles is not increased if the one transferrin variant is excluded from the data base. The number of classes of rare variants detected among the serum proteins was higher than for either erythrocyte proteins or erythrocyte enzymes $\left(\chi^{2}=3 \cdot 2\right.$, D.F. $\left.=1, P<0 \cdot 10\right)$. 
Table 4. Distribution of allele frequencies in the Caucasian sample of Ann Arbor

\begin{tabular}{ccc} 
& \multicolumn{2}{c}{ Number of occurrences } \\
\cline { 2 - 3 } Allele frequency & $<1400^{\mathrm{b}}$ & $>1400^{\mathrm{b}}$ \\
$\leqslant 0.0002$ & 0 & 38 \\
$0.0003-0.0005$ & 1 & 12 \\
$0.0006-0.0010$ & 2 & 4 \\
0.001 I -0.0020 & 3 & 6 \\
0.002 I -0.0050 & 0 & 0 \\
0.005 I -0.0100 & 0 & 4 \\
0.01 I -0.050 & 0 & 4 \\
0.05 I -0.100 & 0 & 1 \\
0.1 I -0.20 & 0 & - \\
$0.21-0.50$ & 2 & 2 \\
$0.51-0.75$ & 2 & 1 \\
$0.76-0.999$ & 3 & 28 \\
I.000 & 8 & 9 \\
a Note that intervals are not equal. \\
Dample size.
\end{tabular}

Table 5. Various comparisons of allele frequency in the Caucasian sample

A. Total variants - classes/1000 alleles Monomers $\quad 0.67$ Polymers $\quad 0.49$

Rare variants - classes/1000 alleles
Monomers
Polymers Polymers $\quad 0.25$

Rare variants - copies $/ 1000$ alleles
Monomers
I.08 Polymers $\quad 0.44$

B. Rare variants - classes $/ 1000$ alleles
Monomorphic
Pon Polymorphic $\quad 0.36$

Rare variants - copies $/ 1000$ alleles
Monomorphic Polymorphic $\quad 1 \cdot 26$

C. Total variants - classes/1000 alleles
Serum proteins Erythrocyte proteins $\quad 0.59$ Erythrocyte enzymes $\quad 0.64$ Rare variants - classes $/ 1000$ alleles
Serum proteins
0.48 Erythrocyte proteins $\quad 0.26$

The relative electrophoretic mobility of the variant classes is symmetrically distributed about the primary isozyme. The charge gain or loss of the variants was estimated from their mobility relative to the separation of the primary isozyme and other variants in the same system and also from the mobility of the degradation products/secondary isozymes relative to the parent molecule. The assignment of relative mobilities is a subjective estimate and is not meant to imply that the actual amino acid composition of the variants is known. Twenty-four allelic subunits have a mobility of plus one charge unit, while 23 are minus one relative to the most common allele. The number of additional alleles which differ by other than one charge unit are: $+2 / 13,+3 / 7,+4 / 3$ and $-2 / 14,-3 / 5,-4 / 3$. Nine other variant subunits had 
Table 6. Relationship of subunit size and variant frequency in the Caucasian sample expressed as classes of variants per 100 amino acid residues

\begin{tabular}{|c|c|c|c|c|}
\hline & & Intercept & Slope & $\begin{array}{c}\text { Correlation } \\
\text { coefficient }\end{array}$ \\
\hline \multirow[t]{3}{*}{ A. } & Rare variants & & & \\
\hline & Monomeric proteins & I.OI & 0.12 & 0.32 \\
\hline & Polymeric proteins & -0.44 & 0.57 & 0.60 \\
\hline \multirow[t]{3}{*}{$B$} & Rare variants & & & \\
\hline & Monomorphic loci & 0.60 & 0.18 & 0.34 \\
\hline & Polymorphic loci & 0.50 & 0.30 & 0.67 \\
\hline ( 1 & Rare variants & 0.58 & 0.22 & 0.42 \\
\hline D. & Total variants & $1 \cdot 48$ & 0.30 & 0.44 \\
\hline
\end{tabular}

intermediate mobilities. Again they were distributed symmetrically about the primary isozyme.

The relationship between subunit size and the number of different variants at a locus is outlined in Table 6 . At all loci where no rare variants had been detected, it was assumed that this reflected sample size limitations rather than an absolute estimate of the frequency. Therefore, a 'calculated' rare variant frequency was obtained for these nonvariant loci, by assuming a numerator of 0.4 variants and a denominator equal to the number of alleles actually studied. a technique similar to that previously employed by Harris et al. (1977). In the few cases for which the subunit size of the various proteins, for the human, was not available from the literature, data from the most closely related species were used. The regression of classes of rare variants (following the adjustment described above) on number of amino acids per peptide was calculated assuming a linear relationship. The data are consistent with the expectation of more variant classes being associated with larger gene products, the scale being approximately $0 \cdot 2$ variant classes for each 100 amino acid residue increase in mass. The slope is steeper, $0 \cdot 3$ variant classes per 100 residues, when the relationship between total variant classes per locus (rare plus polymorphic allele classes) and subunit size is the basis for the calculation. The effect of subunit size on the number of variant classes was more pronounced for polymers than monomers and also for polymorphic over monomorphic proteins.

\section{DISCUSSION}

Only two other studies have examined rare variant frequencies at such a range of loci in similar large, nontribal population surveys. The rare variant frequency in the Japanese study was 1.9/1000 determinations, an allele frequency of 0.00095 (Neel et al. 1978). The variant frequency in similar data from England, following exclusion of the very high frequency for placental alkaline phosphatase variants, is $1 \cdot 1$ heterozyotes/1000 individuals, an allele frequency of 0.00057 (Harris et al. 1974). These figures contrast with estimates of 1.3 rare variant heterozygotes per 1000 determinations (allele frequency of 0.00068) for Ann Arbor Caucasians and $2 \cdot 8$ rare variant heterozygotes per 1000 determinations (allele frequency of 000141) for Ann Arbor Blacks. If, however, the 'adjustment' for possible admixture effects described earlier is omitted, the variant frequency for Ann Arbor Caucasians becomes $2 \cdot 2$ 
variants $/ 1000$ determinations while the variant frequency for Blacks remains about the same at 2.9 variants $/ 1000$ determinations. All 25 of the loci included in the Japanese data base and 23 of the 43 loci in the English study are included in the 51 loci examined in this study (most of the other loci in the English survey are expressed only in tissues other than erythrocytes).

The frequency of different rare variant classes in the English sample is 0.35 different rare variants/1000 determinations (Harris et al. 1974). The comparable figure for Japan is 0.43 rare variant classes/1000 determinations (Neel et al. 1978). The frequency of unique rare variants in the Ann Arbor Caucasian sample is similar, 0.55 classes/ 1000 determinations, but the frequency of 1.87 rare variant classes/1000 determinations in the Black sample is much higher than the other three estimates. The difference in frequency of rare variant classes in Ann Arbor Blacks and Caucasians is increased slightly if the 'adjustment' for admixture is removed.

In all of the groups, approximately $50 \%$ of the rare variants are identified in only a single individual. In the English study, no rare variants were detected at 21 of 43 loci, while five of the remaining loci exhibit only polymorphic variation. Eleven of 25 loci in the Japanese study were without rare variants, while four of the 25 loci exhibited only polymorphic variants. No rare variants were detected at 20 of 51 loci in the Ann Arbor Caucasian sample, although four of the remaining 31 loci did exhibit polymorphic variation. Thus, in each study, over $40 \%$ of the loci did not yield a rare variant even though the number of individuals studied generally ranged from $1000-10000$.

As only four of the rare variants in the Ann Arbor data base (two variants at the PGM2 locus and one each for HGBB and GOTS, see Table $1 a, b$ ) were apparently in common to the two ethnic groups, it would seem unlikely that the higher incidence of rare variants in the Black sample reflects only admixture of the Black gene pool with Caucasian genes. To what extent the increased frequency of variant classes in the Black population is an artifact reflecting the smaller sample size is unclear, although the apparently higher frequency of rare variants is observed in other data where the Black and Caucasian samples are of similar size (Silver, 1982; Gershowitz, pers. commun.).

Previous data, summarized from several different human populations, have indicated that $56 \%$ of monomeric enzymes are polymorphic, while only $30 \%$ of the polymeric proteins are polymorphic (Harris et al. 1977; Ward, 1977). Within the Ann Arbor sample, 34\% of the monomers are polymorphic while $24 \%$ of the polymers are polymorphic. These numbers are not significantly different from the data collected by Harris et al. (1974) in their study of European populations. The reduced proportion of polymorphic loci in the latter two studies probably reflects the fact that the loci included in these studies were selected because of the availability of an electrophoretic technique rather than other selective criteria.

Within the Ann Arbor Caucasian sample, the number of variant classes, both total and rare, and the frequency of rare variant heterozygotes, are higher for monomeric than for polymeric proteins, but the differences are not statistically significant. Similar observations had been noted in previous summaries of collections of data from various studies (Ward, 1977; Harris et al. 1977). Likewise more classes of rare variants and more copies of rare variants are observed at polymorphic loci than at monomorphic loci. This increase is still seen when the loci are further subdivided into monomers and polymers.

Within a subset of this newborn sample, other techniques have been utilized to identify enzyme deficiency and thermostability variants. Enzyme deficiency variants exist at higher 
frequency than do electrophoretic mobility variants in both populations, enzyme deficiency alleles being detected with a frequency of $3 \cdot 1$ variants/1000 determinations in Caucasians and $19 \cdot 2$ variants/ 1000 determinations in Blacks (Mohrenweiser, $1981 ; 1983$; in preparation). The frequency for the Black population is reduced to $9 \cdot 1$ deficiency variants if the polymorphic $\mathrm{TPI}^{*} 1^{\circ}$ and $\mathrm{G} 6 \mathrm{PD}^{*} \mathrm{~A}^{-}$variants are excluded. The allele frequency for thermostability variants not characterized by an altered electrophoretic mobility is 4 variants/1000 determinations in the Caucasian sample (Mohrenweiser \& Neel, 1981). (Data are not available for estimating the frequency of thermostability variants in the Black sample.) The total detectable variation associated with rare variants in the Caucasian population becomes 8-10 variants/1000 determinations if the data from the three approaches, which detect classes of genetic variation which do not overlap significantly, are combined.

The frequency distribution of the relative electrophoretic mobilities of the products of the variant alleles is consistent with previous observations and with models of step-wise mutation (Fuerst \& Ferrell, 1980; Haldorson \& King, 1976; Marshall \& Brown, 1975). It is intriguing to note that 12 of the 18 variant classes which differ from the most common allele by 3 or 4 charge units exist at polymorphic loci, although the polymorphic loci constitute only $\mathbf{1 5}$ of the $\mathbf{5 1}$ loci studied and account for only 30 of the 79 rare variant classes identified. Therefore, the variant frequency at the polymorphic loci would not be the only explanation for this distribution. It is interesting to speculate that intragenic recombinational events as well as recurrent mutation at an already polymorphic allele, may be responsible for generating some of these variants with mobilities that are quite different from the common alleles (Bowman \& Kurosky, 1982; Carter et al. 1979; Takahashi et al. 1982).

A positive relationship between subunit molecular mass and both the number of classes and number of copies of rare variants was detected in this study, the increase being of the order of 0.3 variants for each 100 amino acid residue increase in peptide length and similar to other estimates (Ward, 1978). The relationship is stronger for polymeric proteins than for monomers, as was previously observed (Eanes \& Koehn 1978; Harris et al. 1977; Koehn \& Eanes, 1977). This would be consistent with the larger proteins (genes) presenting a larger target for mutation. It should also be noted that many gene products are identified, following electrophoretic separations, by functional characteristics, thus amino acid substitutions which alter protein function or stability may not be detected as electrophoretic mobility variants. Therefore, the size/variation relationship could also be explained if larger proteins have less constrained conformations and therefore are more capable of tolerating a range of different amino acid substitutions. Also, mutations giving rise to variants with impaired function would often be associated with negative selection and therefore have a greater probability of not being transmitted to future generations. The real explanation for the relationship between subunit size and variant frequency is undoubtly an interplay of both factors but is probably dominated by the conformational requirements for catalytic function and protein stability, as observed with recent studies using site directed mutagenesis techniques and genetic variants (e.g. Daar et al. 1986, Straus et al. 1985).

This work was supported by Department of Energy contract DE-AC02-82ER-60089. Many individuals have provided a tremendous amount of assistance, which is most gratefully acknowledged, during the generation of this data base, the management of the data and the writing of the manuscript. 


\section{REFERENCES}

Asakawa, J. \& Mohrenweiser, H. W. (1982). Characterization of two new electrophoretic variants of human triosephosphate isomerase: stability, kinetic and immunologic properties. Biochem. Genet. 20, 59-76.

Barrantes, R., Smouse, P. E., Neel, J. V., Mohrenweiser, H. W. \& Gershowitz, H. (1982). Migration and genetic infrastructure of the Central American Guaymi and their affinities with other tribal groups. Am.J. Phys. Anthropol. 58, 201-214.

Bowman, B. H. \& Kurosky, A. (1982). Haptoglobin : the evolutionary product of duplication, unequal crossing over, and point mutation. Adv. Hum. Genet. 12, 189-261.

Carter, N. D., West, C. M., Emes, E., Parkin, B. \& Marshall, W. H. (1979). Phosphoglucomutase polymorphism detected by isoelectric focusing: gene frequencies, evolution and linkage. Ann. Hum. Biol. 6, 221-230.

Daar, I. O., Artymiuk, P. J., Phillips, D. C. \& Maquat, L. E. (1986). Human triosephosphate isomerase deficiency: A single amino acid substitution results in a thermolabile enzyme. Proc. Natl. Acad. Sci. USA 83, 7903-7907.

Eanes, W. F. \& Koenn, R. K. (1978). Relationship between subunit size and number of rare electrophoretic alleles in human enzymes. Biochem. Genet. 16, 971-985.

Fuerst, P. A. \& Ferrell, R. E. (1980). The stepwise mutation model: an experimental evaluation utilizing hemoglobin variants. Genetics 94, 185-201.

Fujta, M., Satoh, C., Asakawa, J., Nagahata, Y., Tanaka, Y., Hazama, R. \& Krasteff, T.(1985). Electrophoretic variants of blood proteins in Japanese. VI. Transferrin. Jap. J. Hum. Genet. 30, 191-200.

Hatdorson, L. \& KING, J. H. L. (1976). Unimodality, symmetry and step - state hypothesis of electrophoretic variation in natural populations. J. Mol. Evol. 8, 351-356.

HaRris, H. \& Hopkinson, D. A. (1976). Handbook of Enzyme Electrophoresis in Human Genetics. New York: American Elsevier.

Harris, H., Hopkinson, D. A. \& Edwards, Y.H. (1977). Polymorphism and the subunit structure of enzymes: a contribution to the neutralist-selectionist controversy. Proc. Natl. Acad. Sci. USA 74, 698701 .

Harris, H., Hopkinson, D. A. \& Robson, E. B. (1974). The incidence of rare alleles determining electrophoretic variants: Data on 43 enzyme loci in man. Ann. Hum. Genet. 37, 237-253.

HAUPT, H. \& Böнм, H. (1977) Isolierung und Charakterisierung eines 7S-beta Globulins aus menschlichen Erythrozyten. Blut 35, 229-239.

Konin, R. K. \& Ean es, W. F. (1977). Subunit size and genetic variation of enzymes in natural populations of Drosophila. Theoret. Popul. Biol. 11, 330-341.

Long, J. C., Naidu, J. M., Mohrenweiser, H. W., Gershowitz, H., Johnson, P. L., Wood, J. W. \& Smouse, P. E. (1986). Genetic characterization of Gainj-and Kalam-speaking peoples of Papua New-Guinea. $A m$. $J$. Phys. Anthropol. 70, 75-96.

Marshall, D. R. \& BRown, A. H. D. (1975). The charge state model of protein polymorphism in natural populations. J. Mol. Evol. 6, 149-163.

Mohrenweiser, H. W. \& Decker, R. S. (1982). Identification of several electrophoretic variants of human ceruloplasmin including CP* Michigan, a new polymorphism. Hum. Hered. 32, 369-373.

Mohrenweiser, H. W. \& FIELEk, S. Identification and characterization of a series of rare G6PD variants in a human newborn series. Hum. Genet. (submitted).

Mohrenweiser, H. W., WaDE, P. T. \& Wurzinger, K. H. (1987). Characterization of a series of electrophoretic and enzyme activity variants of human glucose phosphate isomerase. Hum. Genet. 75, 28-31.

Mohrenweiser, H. W. (1981) Frequency of enzyme deficiency variants in erythrocytes from newborn infants. Proc. Natl. Acad. Sci. USA 78, 5046-5050.

Mohrenweiser, H. W. (1983) Enzyme deficiency variants: Frequency and potential significance in human populations. Isozymes: Curr. Topics in Biol. Med. Res. 10, 51-68.

Mohrenweiser, H.W. Functional hemizygosity in the human genome: Direct estimate from twelve erythrocyte enzyme loci. Hum. Genet. (In the Press).

Mohrenweiser, H.W. \& NEEL, J. V. (1981) Frequency of thermostability variants and estimation of the total "rare" variant frequency in human populations. Proc. Natl. Acad. Sci. USA 78, 5729-5733.

Mourant, A. E., Kopeć, A. C. \& Domaniewska-Sobczak, K. (1976). The distribution of human blood groups and other polymorphisms. Oxford Monographs on Medical Genetics. Oxford, London: Oxford University Press.

Naidu, J. M., Mohrenweiser, H. W. \& Neel, J. V. (1985). A sero-biochemical genetic study of Jalari and Brahmin easte populations of Andhra Pradesh, India. Hum. Hered. 35, 148-156.

Neel, J. V., Mohrenweiser, H. W. \& Gershowitz, H. (1987). A pilot study of the use of placental cord blood samples in monitoring for mutational events. Mutat. Research (In the Press).

Neel, J. V., Mohrenweiser, H. W. \& Meisler, M. M. (1980). Rate of spontaneous mutation at human loci encoding protein structure. Proc. Natl. Acad. Sci. USA 77, 6037-6041. 
Neel, J. V., Ueda, N., Satoh, C., Ferrell, R. E., Tanis, R. J. \& Hamilton, H. B. (1978). The frequency in Japanese of genetic variants of 22 proteins. V. Summary and comparison with data on Caucasians from the British Isles. Ann. Hum. Genet. 41, 429-441.

Pearse, A. G. E. (1972). Histochemistry-theoretical and applied. 3rd ed. vol. 2. Baltimore, MD: Williams \& Wilkins Co.

Reed. T. E. (1969). Caucasian genes in American Negroes. Science 165, $762-768$.

Sulver, H. (1982). Probability of inclusion in paternity testing. Arlington, VA: American Association of Blood Banks.

Straus, D., Raines, R., Kawashima, E., Knowles, J. R. \& Gilb ert, W. (1985). Active site of triosephosphate isomerase: In vitro mutagenesis and characterization of an altered enzyme. Proc. Natl. Acad. Sci. USA 82, $2272-2276$.

Takaliashi, N., Negi, J. V., Satoh, C., Niskizaki, J. \& Masunari, N. (1982). A phylogeny for the principal alleles of the human phosphoglucomutase-1 locus. Proc. Natl. Acad. Sci. USA 79, 6636 6640.

Thlss, D., Kopeć, A. C. \& Tills, R. E. (1983). The distribution of human blood groups and other polymorphisms: a supplement. Oxford Monographs on Medical Genetics. Oxford, London: Oxford University Press.

WARD, R. D. (1977). Relationship between enzyme heterozygosity and quaternary structure. Biochem. Genet. $15,123-135$.

WARD, R. D. (1978). Subunit size of enzymes and genetic heterozygosity in vertebrates. Biochem. Genet. 16, 799810 .

Wurzinger, K. H. \& Mohrenweiser, H. W. (1982). Studies on the genetic and non-genetic (physiological) variation of human erythrocyte glutamic oxaloacetic transaminase. Ann. Hum. Genet. 46, 191-201. 